\title{
Égida de una administración de justicia precaria: derecho de acceso a la administración de justicia y pluralismo jurídico débil en Colombia
}

\section{Right of Access to the Administration of Justice and Weak Legal Pluralism in Colombia}

\author{
Luisa Fernanda García Lozano* \\ Ricardo Ramírez Lemo**
}

Fecha de recepción: 01 de septiembre de 2010 Fecha de aprobación: 18 de noviembre de 2010

\section{Resumen}

El presente artículo tiene como fin abordar un extremo del derecho de acceso a la administración de justicia formal. Aquí se analiza cómo en un ambiente de desigualdad y exclusión, y de un aparato de administración de justicia incapaz de satisfacer las demandas de acceso a la justicia de toda la población, el Estado adoptó un modelo de pluralismo jurídico débil como égida que no soluciona tal problemática a cabalidad. Dicho modelo no garantizó el acceso a la administración de justicia formal dando status jurídico a diversos mecanismos alternativos de resolución de conflicto. Pero, por el contrario, evidenció la precariedad del acceso a la administración de justicia formal, sentó bases de exclusión indirecta y no contribuyó a generar legitimidad ni credibilidad en el Estado.

Palabras clave: derecho de acceso a la administración de justicia formal, pluralismo jurídico débil, égida, administración de justicia precaria, legitimidad y credibilidad.

Docente e investigadora, Abogada de la Universidad Santo Tomás, Master in Arts of Sociology of Law, Oñati, International Institute for the Sociology of Law, Milan, University and Basque Country University. Actualmente cuenta con varias investigaciones y publicaciones en temas de sociología jurídica. perteneciente a la línea Derecho y Sociedad de la Facultad de Derecho de la USTA y el grupo de jóvenes investigadores. Correo electrónico: elocuencias@yahoo.com

** Estudiante de derecho de la Universidad Santo Tomás, Monitor de la materia de Sociología Jurídica y coordinador de estudiantes del semillero de investigación "Derecho, Sociedad y Pluralismo Jurídico". 


\begin{abstract}
This article aims to address one end of the right of access to formal justice, here is analyzed how in an environment of inequality, exclusion and justice apparatus unable to meet the demands for access to justice for all the population, the State adopted a model of legal weak pluralism as an aegis that does not solve the entire problem. Actually, this model did not guarantee access to formal justice giving legal status to several alternative mechanisms for conflict resolution, however, it showed the precariousness of access to formal justice, laid the groundwork for indirect exclusion and did not contribute to build legitimacy and credibility in the State
\end{abstract}

Key words: right of access to formal administration of justice, legal weak pluralism, aegis, precarious justice administration, legitimacy and credibility.

\section{INTRODUCCIÓN}

El derecho, al ser uno de los principales canales de diálogo entre las diferentes instituciones y la sociedad para garantizar el orden público manteniendo el control social, adquiere la responsabilidad de generar estructuras, órdenes y herramientas que garanticen la convivencia pacífica de la sociedad en conjunto.

Una de las formas que por excelencia tiende a facilitar la consecución de la paz en la sociedad consiste en la facultad que tiene todo ciudadano de hacer efectivos sus derechos, libertades y garantías, consagrados en la constitución y las leyes, mediante el acceso a la jurisdicción estatal que conlleve a una decisión judicial imparcial respecto de sus peticiones surgidas de su interacción social cotidiana. La armonización de dichos principios produce innumerables beneficios, pues se asegu$\mathrm{ra}$, entre otros, la integridad de un orden político, económico y social justo.

Sin embargo, la consecución de este derecho en Colombia no ha sido logrado a cabalidad, ya que no se contó con herramientas que le otorgasen eficacia. Este escenario produce una situación de verdadera confusión en la estructura institucional, generadora de ilegitimidad de la administración de justicia formal con respecto a los ciudadanos.
Lo anterior, entre otras causas, porque Colombia se halla inmersa en contextos de anomia y conflicto, caracterizados por la fragilidad del orden público, donde la miseria y la exclusión, productos del conflicto armado, del narcotráfico y de la corrupción generalizada de las instituciones estatales, afectan seriamente el tejido social.

Ahora bien, en el contexto ya descrito se hace posible identificar dos elementos teóricos de estudio para entender las problemáticas que se afrontan en la práctica y que evidencian las trasformaciones vividas en el último siglo el arribo de corrientes reformistas en el pensamiento jurídico. A saber, la preocupación por el derecho de acceso a la administración de justicia y la formulación de nuevas posturas teóricas como el denominado jurídico.

Aunque existe una multiplicidad de caminos para visualizar la relación que puede existir entre los anteriores conceptos, se partirá de la siguiente hipótesis: la implementación de mecanismos alternativos de solución de conflictos (entendidos como pluralismo jurídico débil), a fin de mitigar la crisis de acceso a la administración de justicia formal y fortalecer tanto su legitimidad como su credibilidad, no ha producido los resultados esperados, pues termina chocando con la marginalización y exclusión de amplios sectores de la población que no acceden al aparato de justicia estatal formal no 
contribuyendo, por tanto, a la credibilidad y legitimidad del aparato de justicia.

La metodología para desarrollar el presente artículo fue la documental, complementada con investigaciones empíricas realizadas, entre otros, por uno de los autores antes del 2003.

El presente texto se desarrollará en tres partes. En la primera de ellos se analizará el derecho de acceso a la administración de justicia, su importancia en el sistema jurídico y en los contextos sociales como medio para lograr los fines esenciales del Estado. En la segunda se estudiarán los mecanismos alternativos de solución de conflictos como expresión de pluralismo jurídico débil, del que no ha podido satisfacer efectivamente las demandas de justicia para muchos sectores de la población.

En la última parte, como conclusión, se establece que la inclusión de nuevos mecanismos que intentan equipararse con los propios de la administración de justicia estatal no se han traducido en mayor acceso a la administración de justicia formal, por lo cual se dejará de manifiesto la necesidad de centrar los esfuerzos del Estado y de la sociedad en la ampliación del acceso a la administración de justicia estatal más que en la ampliación de los mecanismos alternativos si se quiere cumplir con el propósito estatal de garantizar a todos los habitantes una adecuada administración de justicia como medio para lograr un orden público y justa credibilidad y legitimidad del propio Estado.

\section{EL DERECHO DE ACCESO A LA ADMINISTRACIÓN DE JUSTICIA COMO MEDIO PARA EL CUMPLIMIENTO DE LOS FINES DEL ESTADO}

La Constitución Política de 1991 introdujo un derecho fundamental del cual deben gozar todas las personas en el territorio nacional, así: "Se garantiza el derecho de toda persona a acceder a la administración de justicia" (Constitución Política de Colombia, título VIII, capítulo 1, art. 229).

En un sentido filosófico y político, este derecho ha sido catalogado efectivamente como derecho fundamental mediante reiterada jurisprudencia de la Corte Constitucional. En efecto, ésta ha sostenido:

\begin{abstract}
El acceso a la administración de justicia se constituye para el individuo en una necesidad inherente a su condición y naturaleza, sin él los sujetos y la sociedad misma no podrían desarrollarse y carecerían de un instrumento esencial para garantizar su convivencia armónica, como es la aplicación oportuna y eficaz del ordenamiento jurídico que rige a la sociedad, y se daría paso a la primacía del interés particular sobre el general, contrariando postulados básicos del modelo de organización jurídica-política por el cual optó el Constituyente de 1991. Así, el acceso a la administración de justicia se erige en nuestro ordenamiento superior como un derecho fundamental de los individuos, que como tal prevalece y goza de protección especial por parte del Estado (Corte Constitucional, Sentencia T-476/98 M.P. Fabio Morón Díaz).
\end{abstract}

A su vez, algunos autores sostienen que este derecho puede ser definido como:

Una forma de materializar los principios constitucionales mediante la garantía y ejercicio de los derechos fundamentales y como parámetro para establecer la legitimidad del Estado; se consolida como pilar del modelo de Estado acogido por la carta y del derecho que permite el acopio de los planteamientos jurídicos y políticos realizados, dado que es un mandato social esencial y primordial para un sistema legal que pretenda garantizar los derechos tanto individuales como colectivos (Fajardo, et ál., 2006, p. 36).

Ahora, como visión instrumental que se relaciona directamente con su efectividad, expone el antes citado tribunal la manera de su consecución: 
[...] cuando, dentro de determinadas circunstancias y con arreglo a la ley, el juez garantiza igualdad a las partes, analiza las pruebas, llega a un libre convencimiento, aplica la Constitución y la Ley y, si es el caso, proclama la vigencia y realización de los derechos amenazados o vulnerados. Es dentro de este marco que la Corte Constitucional no ha vacilado en calificar al derecho al que hace alusión la norma que se revisa -que está contenida en los artículos 29 y 229 de la Carta Políticacomo uno de los derechos fundamentales [...] (Corte Constitucional, Sentencia T-006/92 M.P. Eduardo Cifuentes Muñoz).

La anterior significación se incluye dentro de una definición de administración de justicia formal, en tanto parte de la base de un sometimiento al imperio de la ley y la garantía de igualdad a las partes que, entre otras, debe garantizar la similitud en el procedimiento que ha sido preestablecido $y$ desarrollado por la Constitución y la ley.

Con todo, la materialización de dicho derecho ha sido obstruida frecuentemente, lo cual denuncia la precariedad del sistema. Entre las principales causas se encuentran: los costo de los litigio, las reclamaciones pequeñas y los pequeños litigios que cogestionan el aparato de justicia, el tiempo excesivo de los trámites procesales; la cogestión y retardos de los despachos judiciales y la desigualdad social (Rodríguez, 2001 \& De Sousa, et ál., 2001). A lo cual podemos agregar, sin duda alguna, la situación del conflicto armado que dificulta el acceso a la justicia en aquellas zonas afectadas por él, pues: "[...] la presencia de actores armados incide negativamente en el número de los casos que reciben y deciden los jueces [...]" (García, 2008, p. 189).

Entrando en el detalle podemos individualizar las principales problemáticas de la siguiente forma:

1. Un aparato estatal que es incapaz de sobrellevar la carga de litigiosidad que produce el país: el principal argumento para esta crisis es el de casos para ser conocidos por el sistema en las últimas décadas, por ejemplo, "En Colombia, la demanda por cien mil habitantes ante la jurisdicción ordinaria casi se duplica entre 1993 y 2000 [...] y los egresos no logran superar el $90 \%$ de las demandas [...]" (FixFierro, 2006, p. 18).

2. Factores procedimentales y trámites burocráticos: la duración de los procedimientos sobrepasa, en la mayoría de los casos, los términos procesales, lo cual conduce a la situación de Ios procesos: en Colombia, de acuerdo a una investigación del Instituto Ser de Investigación, en 1994 tenían más de 2.573.406 de casos represados en el sistema judicial, los cuales se mantenían para 1997. Pero habían aumentado por encima de cuatro millones de procesos (Herrera, 2007).

3. Costos del litigio: aunque en principio el servicio de administración de justicia es gratuito, las expensas procesales y los trámites en general, implican gastos (recursos económicos) que amplios sectores sociales no pueden sufragar.

4. Conflicto armado: no basta con instituir formalmente juzgados en ciertos sectores de la población, pues sin acompañamiento institucional la población y los órganos de justicia pueden verse amedrentados por los actores en conflicto, lo cual dificulta el acceso al aparato, pues los jueces terminan por convertirse en: "[...] nominales a quienes les llega sólo una pequeña parte de la conflictividad social; una parte que ha sido filtrada previamente por los actores armados" (García, 2008, p. 194).

Los aspectos enunciados configuran algunas circunstancias que visibilizan los impedimentos de amplios sectores de la población para acceder a la justicia estatal. La precariedad aquí radicaría directamente en la imposibilidad de acceder al despacho judicial, más allá de la forma como sea 
fallado el caso por el juez. Ese es un aspecto muy importante para el análisis, puesto que entre las principales fórmulas de solución, el Estado colombiano estableció figuras o mecanismos que tienden a sobrellevar dicha crisis de acceso.

En virtud de las anteriores acotaciones, cabe dar un breve repaso a las reformas auspiciadas por los Estados Unidos en Colombia, principalmente, que han buscado estimular el uso de mecanismos alternativos de solución de conflictos (MASC). Las primeras iniciativas se sitúan en la década del sesenta, las cuales tenían como objetivo el desmonte del constitucionalismo social, el énfasis en el rol de la justicia como facilitadora del mercado y la apertura de la administración de justicia a criterios de eficacia del mercado (Rodríguez \& Uprimny, 2003; Domingo \& Sieder, 2001).

En este orden de ideas, la modernización de la enseñanza del Derecho (Rodríguez, 2000, p. 20) fue el primer gran objetivo, impulsado por la Agencia de los Estados Unidos para el Desarrollo Internacional (USAID, por sus siglas en inglés) y la Fundación Ford. Aquí se buscó una reforma educativa que permitiera la implantación de los principios y la metodología jurídica norteamericana liberal. De igual manera, se apoyaron los "Servicios jurídicos gratuitos de asesoría legal, amparo de pobreza y defensores de oficio" (Corte Constitucional, Sentencia C-195 de 2001 M.P. Manuel José Cepeda Espinosa y Marco Gerardo Monroy Cabra).

Las reformas se llevaron a cabo principalmente en las Universidades de Colombia, Chile y Brasil. A mediados de 1970 se valoraron los programas y la conclusión fue que el modelo había fracasado por varias razones:

En primer lugar: los proyectos fueron etnocéntricos e ingenuos en tanto acogieron incondicionalmente el modelo estadounidense de enseñanza del derecho y buscaron trasplantarlo a América Latina sin tener en cuenta la cultura jurídica y las condiciones sociales

\begin{abstract}
locales. En segundo lugar, chocaron con los poderosos intereses protegidos por el sistema prevalente en América Latina, dado que dicho sistema les permitía a los profesores y estudiantes no dedicarles el tiempo completo a sus actividades académicas y era económico para las facultades de derecho (Rodríguez, 2000, p. 24).
\end{abstract}

En 1980 comienza un segundo bloque de proyectos y programas con énfasis en el desarrollo y la función del derecho en el progreso económico, los cuales fueron auspiciados por USAID. De igual forma, la Fundación Ford apoyó algunas organizaciones de derechos humanos, en los procesos de retorno a la democracia tras las dictaduras en el cono sur.

\begin{abstract}
A partir de los años noventa, el Banco Interamericano de Desarrollo y el Banco Mundial se suman a la tarea y adoptan estrategias desde un enfoque sistémico, intentando trabajar simultáneamente en varios aspectos de la organización judicial, procedimientos, leyes y vínculos externos (Hammergren, 1999, p. 12).
\end{abstract}

Las reformas de esta última generación se centran en un desarrollo eficientista e institucional, motivo por el cual se inicia la implementación de Ios MASC, al constituir estos una posibilidad de disminuir las cifras de procesos en los tribunales, al encargarse de casos de menor cuantía que implicaban un represamiento en el aparato de administración de justicia.

\section{PLURALISMO JURÍDICO DÉBIL: ÉGIDA DE UNA ADMINISTRACIÓN DE JUSTICIA PRECARIA}

Pasando al campo teórico, conviene encuadrar lo reseñado anteriormente en una teoría que tienda a entender y evaluar dichas estrategias. De esta manera, el pluralismo jurídico se presenta como llave idónea para tal fin, pues representa un cambio en la forma de pensar y concebir el Derecho desde una perspectiva en la que se da más prevalencia a la realidad que a la ficción o ideal dogmático. 
pluralismo jurídico es concebido como "una situación en la cual uno o más sistemas legales coexisten en el mismo campo social" ${ }^{\prime 3}$ (Engle, 1980, p. 870). Una de las hipótesis sostenidas por diversos autores, ha nacido gracias a un mosaico de:

\section{[...] fenómenos que han sido atribuidos a la crisis del estado-nación: por un lado, por el surgimiento de viejas y nuevas entidades colectivas y reclamos de reco- nocimiento; por el otro, por la imposición de modelos y relaciones económicas, que a su vez generan modelos jurídicos que traspasan los confines nacionales, muchos de estos fenómenos originan normas infra, supra, y trasnacionales, que no se identifican con los ordena- mientos estatales (Facchi, 2005, p.31).}

Empero, el tema de primordial interés para el presente escrito lo constituye la teoría del pluralismo jurídico débil. Esta teoría es desarrollada por el profesor Jhon Griffiths, quien parte como contraposición necesaria, de la descripción del centralismo jurídico como aquel en donde: "El Derecho es y debe ser el Derecho del Estado, uniforme para todas las personas, con exclusión de cualquier otra forma de Derecho y administrado por un único conjunto de instituciones del Estado"4 (Griffiths, 1986, p. 3).

Además, señala este profesor que:

Una situación de pluralismo jurídico -la omnipresente y normal situación en la sociedad humana- es una en la cual el derecho y las instituciones jurídicas no son subsumibles todas dentro de un "sistema" pero tienen sus fuentes en las actividades auto-reguladoras de todos los diversos campos sociales presentes [... $]^{5}$ (Griffiths, 1986, p. 39).
Es por esto que al detenerse a desenmascarar el pluralismo jurídico débil, no duda en explicar:

En este sentido (débil) un sistema jurídico es "plural" cuando el soberano (implícitamente) ordena (o la norma fundamental da validez, y así sucesivamente) diferentes organismos de derecho para diferentes grupos en la población ${ }^{6}$ (Griffiths, 1986, p. 5).

La importancia que tiene deslindar la concepción de pluralismo jurídico fuerte de su disímil débil, nace de un aspecto político:

En el sentido débil -La posible designación de una política pública legal dentro del discurso interno del derecho estatal - solamente se asemeja con el pluralismo jurídico por la confusión nominal como la designación de un estado empírico de situaciones en la sociedad ${ }^{7}$ (Griffiths, 1986, p. 8).

Es decir, en el pluralismo jurídico débil simplemente se da cabida, dentro del discurso centralista estatal, al reconocimiento de situaciones o prácticas que, generalmente, están presentes en la sociedad, otorgándoles reconocimiento legal. Lo que en últimas es subsumido en el Estado centralista, sin brindarles plena autonomía pero con la apariencia de tal.

Es de esta manera como el Estado permite el uso de prácticas paralelas simultáneas, con miras a la resolución de conflictos, siempre con su aval y bajo su monopolio; sin reconocer, desde luego, la existencia de diferentes y autónomas órdenes legales en un mismo espacio-tiempo.

3 "A situation in which two or more legal system coexist in the same social field". En el idioma original del autor.

4 "law is and should be the law of the state, uniform for all persons, exclusive of all other law and administered by a single set of state institutions". En el idioma original del autor.

5 "a situation of legal pluralism -the omnipresent, normal situation in human society- is one in which law and legal institutions are all not subsumable whitin one 'system' but have their sources in the self-regulatory activities of all the multifarious social field present, [...]". En el idioma original del autor.

6 "In this ('Weak') sense a legal system is 'pluralistic' when the sovereign (implicitly) commands (or the grundnorm validates, and so on) different bodies of law for different groups in the population". En el idioma original del autor.

7 "In the weak sense - the designation possible legal policy within the internal discourse of state law - has only confusion nominal resemblance to legal pluralism as the designation of an empirical state of affairs in society". En el idioma original del autor. 
Esta flexibilización del monopolio para impartir justicia ha sido calificada por algunos autores como alternativa, sobre todo en el caso colombiano, donde dichos mecanismos aceptados por el Estado, representan procesos de institucionalización con diferentes intensidades. Por eso, al hablar de formas alternativas de solucionar conflictos se señala:

[...] la creciente significación de determinados procedimientos legales que son tolerados, admitidos o inclusive institucionalizados, que pueden contribuir, sino del todo, por lo menos parcialmente, como canales que viabilizan prácticas jurídicas más ágiles y flexibles (Wolkmer, 2006, p. 251). Punto éste de vital importancia, pues como pasará a demostrarse, gracias a estos mecanismos el Estado busca revestirse de credibilidad y legitimidad.

Antes de seguir adelante, cabe anotar que figuras como la conciliación en equidad, la mediación y el arbitramento (implementados gracias a la Ley 23 de 1991), hacen parte de los MASC, no así la jurisdicción especial de paz, que es reconocida como administración de justicia por el artículo 247 de la Carta Política. Pero, para lo anotado en este artículo, representa aquella forma alterna de impartir justicia, validada por el Estado, lo hace parte del pluralismo jurídico en sentido débil.

El caso especial de los jueces de paz, aunque son reconocidos como una forma de acceso a la justicia estatal (pues se hayan reconocidos por la Constitución y la ley como jurisdicción), no son administración de justicia formal en el sentido de estar sometidos y guiados directamente por el andamiaje jurídico, pues aquellos son:

\footnotetext{
[...] líderes comunitarios dotados de cualidades humanas y sensibilidad social y comunitaria; ciudadanos en ejercicio postulados por organizaciones comunitarias con personería jurídica o grupos organizados de vecinos para ser elegidos popularmente, que tienen la facultad de administrar justicia, basados en la equidad o justo comunitario y no en la ley preestablecida (Ariza \& Abondano, 2009, p. 16).
}

Con lo que se incluyen dentro de una forma alterna a la justicia formal para resolver conflictos.

Para reafirmar lo anterior, señalan críticamente los mismos autores que la Jurisdicción Especial de Paz representa: "[...] un modelo de justicia que no sea el adjudicativo y formalista que tenemos hegemónicamente desde el positivismo jurídico (el llamado formalismo)" (Ariza \& Abondano 2009, p. 58).

Es por esto que en las anteriores instituciones citadas, la Constitución y la ley reconocen y fomentan prácticas paralelas para la resolución de conflictos sin acudir a la administración de justicia estatal formal. No obstante, no les permite manejarse a su arbitrio, pues deben ceñirse a las prerrogativas legales, constitucionales e incluso jurisprudenciales propias del Estado una prueba más de pluralismo jurídico en sentido débil.

En este punto ya puede hablarse de la pretendida égida, entendida como escudo, protección o defensa. Pues, tratándose de los MASC y de la Jurisdicción Especial de Paz como manifestación de pluralismo jurídico débil; a manera de ejemplo clarificador, la Corte Constitucional colombiana (en nombre del Estado) establece al menos cuatro de sus fines primordiales a saber:

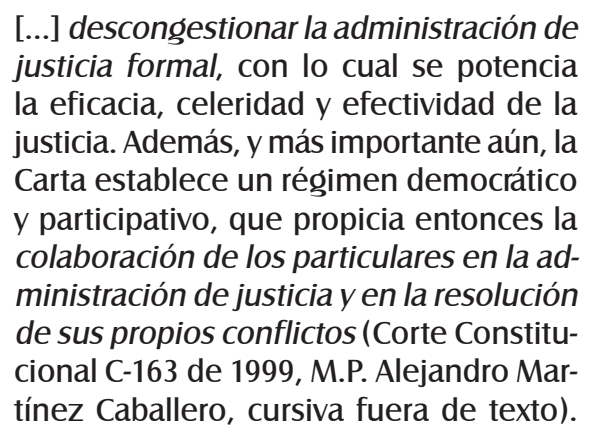

Pero hasta aquí nada parece merecer sospecha, pues la descongestión y colaboración de la ciudadanía, así como la resolución de sus conflictos en un escenario democrático puede sostenerse sin mayor reproche (aún cuando no parezca convin- 
cente su correlación). Sin embargo, es principalmente en la cuarta finalidad en la que se debe ser suspicaz, pues para la administración de justicia colombiana, los MASC:

\section{[...] generan espacios de intervención y desarrollo de la función jurisdiccional evi- tando la conflictivización de la sociedad y logrando, por ende, el fortalecimiento de la legitimidad del aparato de justicia estatal en la medida en que éste puede dedicarse a resolver aquellos asuntos que son de verdadera trascendencia social (Corte Constitucional, Sentencia C-893 de 2001. M.P Clara Inés Vargas Hernández, cursiva fuera de texto).}

He aquí la égida: el aparato de justicia se escuda en los MASC que, principalmente (aunque para sus efectos debe ser sumada la Jurisdicción Especial de Paz), descongestionan el aparato estatal y fomentan la participación democrática, disimulando la precariedad de la administración de justicia formal y reclamando luego, para sí misma, la legitimidad y credibilidad que aquellos crean, dejando en un segundo plano la problemática real del acceso a la administración de justicia formal.

Es así como el Estado extrae, desde luego, una maravillosa virtud del pluralismo jurídico débil a favor de la administración de justicia formal. Sin embargo, lo que diga la letra no siempre es fiel con lo que presenta la realidad, pues tal pretendida legitimidad en su favor puede no ser verdadera $y$ sí, en cambio, puede distanciarla de dicho objetivo.

\section{A modo de conclusión}

\section{La inclusión de nuevos factores no altera el resultado}

Cabe recordar lo dicho por la misma Corte Constitucional:

El artículo 229 superior reconoce a todas las personas el derecho a obtener tutela judicial efectiva por parte de los jueces y tribunales que integran la administra- ción de justicia, garantía que entraña la posibilidad de acudir libremente a la jurisdicción siendo parte en un proceso promoviendo la actividad jurisdiccional [...] (Corte Constitucional, Sentencia C-893 de 2001, M.P. Clara Inés Vargas Hernández).

Sin embargo, garantizar el derecho de libre acceso a la jurisdicción no tiene sentido si tan sólo se pueden ofrecer plenamente los servicios de administración de justicia informal, denegando a la formal estatal, pues el mero hecho de solucionar conflictos no garantiza el acceso a la administración de justicia formal, tan sólo aliviana la carga que debió remediar de modo efectivo.

Por eso, aunque en virtud del modelo de pluralismo jurídico débil se incluyen nuevos mecanismos y jurisdicciones, que entre otras características tienden a descongestionar el aparato de justicia para que éste se pueda dedicar a asuntos de "verdadera trascendencia" (como señaló la Corte Constitucional), no se está apuntando al punto de los resultados esperados, el cual es facilitar el acceso a la administración de justicia formal.

Si bien es cierto, al disminuir la carga de procesos en los despachos judiciales se da la posibilidad de que nuevos asuntos accedan a la justicia formal, ¿qué pasa con quienes acudieron a la informal? Ellos no accedieron a la administración de justicia formal, por el contrario, acudieron y además (como punto importante) comenzaron a legitimar $y$ a dar credibilidad a otros modelos.

Este golpe en contra de la justicia formal se manifestaría en aquellos sectores de la sociedad que sientan que la administración de aquella es precaria, dado que se ve obligada a ofrecer otros caminos (cerrar la puerta indirectamente) a asuntos que no representan "mayor trascendencia", es decir, a sus asuntos. Mientras que los MASC y los jueces de paz dan trámite pronto y eficaz a sus conflictos, lo que reviste a estos últimos de confianza, credibilidad y lo más importante, legitimidad. 
Así se plantea un escenario de exclusión indirecta e incluso voluntaria (si se quiere aceptar la tesis de libre acceso a la jurisdicción) de acceso a la administración de justicia formal, pues en vez de suministrarse mayor cobertura y oferta de acceso, a éste se ofrecen caminos alternos a aquella.

\section{¿Ampliación de la égida o del derecho de acceso?}

La participación de la sociedad civil en los asuntos que la afectan, aunque sea un fin loable, no garantiza el libre acceso a la administración de justicia formal. Mucho menos puede pretenderse que el Estado gane legitimidad arrojando a sectores de la sociedad civil a mecanismos alternativos de solución de conflictos. Es cierto que amaina el descontento general respecto de la solución de sus controversias, pero esto no gracias a la administración de justicia formal, pues quienes realmente ganan credibilidad y legitimidad son los MASC junto a los jueces de paz.

Es por esto que si lo que se quiere es superar la precariedad del aparato de administración de justicia en cuanto a la capacidad de acceso a éste, la solución no reposa en crear una égida que disminuya la carga de litigios, ya que en esta forma no se afronta la problemática directamente, sino que se desvía y descarga en otros sistemas alternativos.

Ahora bien, si lo que se quiere fortalecer es el acceso a la administración de justicia formal y ganar a su vez legitimidad, la solución no está en el fomento del pluralismo jurídico débil. Tampoco se quiere decir con lo anterior que deban desaparecer los MASC y los jueces de paz, sin duda son interesantes herramientas con sus propios ámbitos de desarrollo.

Lo que parece necesario, en aras de ampliar el derecho de acceso a la administración de justicia formal y con ello fortalecer su legitimidad, sería el superar la precariedad del aparato mismo, mediante la ampliación del presupuesto para la administración de justicia, y con ello, la ampliación de los despachos judiciales. Además debería darse mayor acompañamiento institucional a la justicia en zonas de conflicto armado; procurar el blindaje frente a la corrupción y el narcotráfico; garantizar de forma efectiva la independencia a los órganos judiciales, lo mismo que de políticas públicas que tiendan a ampliar la oferta de acceso a la administración de justicia (entre otras tantas estrategias). Sin embargo, tal formulación de estrategias dedicaría un análisis y estudio mucho mayor que, por su parte, el presente escrito no pretende ni se ve en posibilidad de abordar en esta ocasión.

\section{REFERENCIAS}

Ariza; Rosembert \& Abondano, D. (2009). Jueces de paz. El dilema de lo justo. Bogotá: Universidad Santo Tomás.

Domingo, P. \& Sieder, R. (Eds.) (2001). Rule of Law in Latin America: the International Promotion of Judicial Reform. London: Institute of Latin American Studies, University of London.

Boaventura, S. \& García, M. (Eds.) (2001). El caleidoscopio de las justicias en Colombia: análisis sociojurídico, Tomo I. Bogotá: Siglo del Hombre Editores, Universidad de los Andes, Colciencias, Instituto Colombiano de Antropología e Historia y Universidad Nacional de Colombia.

Boaventura, S. \& García, M. (Coords.) (2004). Democratizar la democracia los caminos de la democracia participativa. México: Fondo de Cultura Económica.

Boaventura, S. \& García, M. (1998). La globalización del derecho. Los nuevos caminos de la regulación y la emancipación. Bogotá: Universidad Nacional de Colombia, Facultad de Derecho, Ciencias Políticas y Sociales e Instituto Latinoamericano de Servicios Legales Alternativos -ILSA. 
Boaventura, S. \& García, M. (1991). Estado, derecho y luchas sociales. Bogotá: Instituto Latinoamericano de Servicios Legales Alternativos - ILSA.

Engle S. (1998). Legal Pluralism, In: Law and Society Review, 22, Massachusetts: Blackwell Publishing.

Facchi, A. (2005). Los derechos en la Europa multicultural, pluralismo normativo e inmigración. Buenos Aires: Universidad de Buenos Aires - Facultad de Derecho.

Fajardo, L., et ál. (2006). Los invisibles y la lucha por el derecho en Colombia. Una mirada desde el Programa Nacional de Casas de Justicia. Bogotá: Universidad Santo Tomás.

Fix-Fierro, H. (2006). Tribunales, justicia y eficiencia. Estudio socio-jurídico sobre la racionalidad económica en la función judicial. México: Universidad Autónoma de México.

García, M. (Ed.).(2008). Jueces sin Estado, la justicia colombiana en zonas de conflicto armado. Bogotá: Siglo del Hombre Editores, Fundación Konrad Adenauer y The John Merck Fund.

Hammerrgren, L. (1999). Quince años de reforma judicial en América Latina: dónde estamos y por qué no hemos progresado más. En: Reforma Judicial en América Latina: una tarea inconclusa. Bogotá: Corporación Excelencia en la Justicia.

Hardt, M. \& Negri, A. (2004). Multitud: guerra y democracia en la era del imperio. Barcelona: Paidós.

Huber, R., et ál. (2008). Hacia sistemas jurídicos plurales. Reflexiones y experiencias de coordinación entre el derecho estatal y el derecho indígena. Bogotá: Konrad Adenauer Stiftung.

Mejía, O. (2002). La Constitución de 1991 como proyecto inacabado. En: El Otro Derecho, 28 (junio - diciembre). Otras miradas de la justicia. Bogotá:
Instituto Latinoamericano de Servicios Legales Alternativos.

Rodríguez, C. \& Uprimny, R. (2003). ijusticia para todos o seguridad para el mercado? En: Restrepo D. (Ed.). La falacia neoliberal crítica y alternativas. Bogotá: Universidad Nacional de Colombia.

Rodríguez, C., García, M. \& Uprimny, R. (2003). Justice and Society in Colombia: A Sociolegal Analysis of Colombian Courts. In: Perdomo, R. \& Friedman, L. (Eds.). Latin legal Cultures in the Age of Globalization. California: Standford University Press.

Von Benda-Beckmann, F. \& Keebet. (2006). The Dynamics of Change and Continuity in Plural Legal Orders. In: Journal of Legal Pluralism and Unofficial Law, 53-54. Berlin: Lit Verlag.

\section{Sentencias}

Colombia, Corte Constitucional, Sentencia T-006 de 1992. Magistrado Ponente: Eduardo Cifuentes Muñoz.

Colombia, Corte Constitucional, Sentencia T-476 de 1998. Magistrado Ponente: Fabio Morón Díaz.

Colombia, Corte Constitucional, Sentencia C-163 de 1999. Magistrado Ponente: Alejandro Martínez Caballero.

Colombia, Corte Constitucional, Sentencia C-893 de 2001. Magistrada Ponente: Clara Inés Vargas Hernández.

Colombia, Corte Constitucional, Sentencia C-1195 de 2001. Magistrados Ponentes: Manuel José Cepeda Espinosa y Marco Gerardo Monroy Cabra.

\section{Páginas web}

Abondano, D. \& García, L. (2004). Conciliación en equidad: ijusticia real?, Trabajo de grado (Abogado). Universidad Santo Tomás - Facultad de De- 
recho, Bogotá. Recuperado de [http://unicornio. usta.edu.co/CONTENIDO/MASCDIG/conciliacionenequidad.pdf].

Corporación Excelencia en la Justicia. (2010). Expedientes represados en la justicia ordinaria, fuente, Consejo Superior de la Judicatura. Recuperado el 1 de noviembre de 2010 de [http://www.cej.org. co/justiciometros/2023-expedientes-represadosen-la-justicia-ordinaria].
Griffiths, J. (1986). What is legal pluralism? In: Journal Of Legal Pluralism, 24. Recuperado el 20 de octubre de 2010 de [http://keur.eldoc.ub.rug.nl/ FILES/wetenschappers/2/11886/11886.pdf].

Herrera, H. (2007). Estado de métodos alternativos de solución de conflictos en Colombia. Recuperado en mayo de 2007 de [www.oas.org/juridico/ spanish/adjusti6.htm]. 